\title{
Tracking control in billiards using mirrors without smoke, Part I: Lyapunov-based local tracking in polyhedral regions
}

\author{
F. Forni, A.R. Teel, L. Zaccarian
}

\begin{abstract}
In this paper we formulate the tracking problem of a translating mass in a polyhedral billiard as a stabilization problem for a suitable set. Due to the discontinuous dynamics arising from nonsmooth impacts, the tracking problem is formulated within a hybrid systems framework and a Lyapunov function is given, which decreases during flow (continuous motion) and remains constant across jumps (impacts of the masses). To guarantee non-increase of the Lyapunov function at jumps, we introduce a novel concept of mirrored images of the target mass and prove that, with this concept, local tracking is achieved. Several simulations illustrate the effectiveness of the proposed approach as compared to alternative solutions. In a companion paper [6] we address global results and generalize the local approach to curved billiards.
\end{abstract}

\section{INTRODUCTION}

Control of dynamical systems subject to nonsmooth impacts is a relevant problem in several application areas, often related to the robotics field [2]. Several Lyapunov-based solutions to the stabilization and tracking problem of systems with nonsmooth impacts have been proposed in the past decade [3], [10], [20]. Some of them address the problem via the larger class of complementarity Lagrangian systems. See [13] for a recent work which gives an updated overview of the results in this fields and generalizes and improves the results in the previous papers [1], [4]. Several additional recent techniques addressing tracking control with impacts both from a theoretical and an experimental viewpoint are provided in the works [14], [15], [11], [12], [19] and references therein. The reader is referred to [13] for a more detailed overview.

Tracking control in billiards is a representative example of the control problem discussed above whenever the control action is allowed to act during the motion (like, e.g, in walking robots) and the impacts correspond to jumps in the state occurring whenever the trajectory reaches a constraint. In this context, a number of results have been produced, which rely on the model first proposed in [20]. These are nicely summarized in [7],[12]. The parallel problem of tracking trajectories while restricting the control action at the impact times is addressed in the work of [16] and references therein.

The problem statement in this paper is motivated by [7], [12] where Lyapunov-based tracking control is designed for

Research supported in part by AFOSR grant number FA9550-09-1-0203, NSF under grants ECCS-0925637 and CNS-0720842, by ENEA-Euratom and MIUR.

F. Forni and L. Zaccarian are with the Dipartimento di Informatica, Sistemi e Produzione, University of Rome Tor Vergata, 00133, Italy, forni@disp.uniroma2.it, zack@disp.uniroma2.it, A.R. Teel is with the Department of Electrical and Computer Engineering, University of California Santa Barbara, CA 93106-9560, USA, teel@ece.ucsb.edu. a mass moving on a billiard. We cast this problem within the framework of [9], [8] for hybrid dynamical systems and propose a novel control strategy which is capable of inducing decrease of a suitable Lyapunov function during flows and non-increase during jumps. This type of approach is somewhat new in the area as most of the existing Lyapunov-based results treat the impacts as events which locally increase the Lyapunov function (a notable exception being the case in [14]) and resort to weak stability concepts. Here, instead, we design a Lyapunov function (and the arising control law) which does not increase at the impact times because it computes the tracking error based on a mirrored image of the reference, whenever this is convenient to keep the tracking error small. The resulting controller selects which mass to track based on the closest reference among all the possible reflections given by the billiard boundaries. It is notable that a so-called "mirror algorithm" is proposed in [5] to solve the juggling problem. This has little similarity with our approach as the "mirror" is used there at all times to make the actuator track a mirror image of the ball trajectory (which is regulated acting at jumps), whereas here it is used to prevent the tracking algorithm (which acts during flows) from getting confused by impacts. Local tracking for polyhedral billiards is discussed here and further developments are given in the companion paper [6], where global tracking for some special cases is provided and local tracking in curved billiards is also achieved.

The paper is structured as follows. Section II introduces the proposed dynamical model. In Section III local tracking with one boundary is discussed. In Section IV the results are extended to the case of multiple boundaries and an example is discussed in Section V.

Notation: The Euclidean norm of a vector is denoted by $|\cdot|$. The distance between two sets $S_{1}$ and $S_{2}$ is given by $\inf _{s_{1} \in S_{1}, s_{2} \in S_{2}}\left|s_{1}-s_{2}\right|$ and it is denoted by $d\left(S_{1}, S_{2}\right)$. The distance between a point $s$ and a set $S$ is denoted by $|s|_{S}$ and it is equal to $d(\{s\}, S)$. A continuous function $\alpha(\cdot)$ : $[0, a) \rightarrow[0,+\infty)$ is said to belong to class $\mathcal{K}$ if it is strictly increasing and $\alpha(0)=0$; it is said to belong to class $\mathcal{K}_{\infty}$ if $a=+\infty$ and $\lim _{r \rightarrow+\infty} \alpha(r)=+\infty$. Given a vector $z \in \mathbb{R}^{4}$, we will consider $z=\left[\begin{array}{ll}z_{p}^{T} & z_{v}^{T}\end{array}\right]^{T}$ where the subvectors $z_{p}, z_{v}$ belongs to $\mathbb{R}^{2}$. For any given function $V: \mathbb{R}^{n} \rightarrow \mathbb{R}, \nabla V$ is the vector $\left[\begin{array}{lll}\frac{\partial V}{\partial x_{1}} & \ldots & \frac{\partial V}{\partial x_{n}}\end{array}\right]^{T} \cdot\left\langle v_{1}, v_{2}\right\rangle$ denotes the scalar product between the vectors $v_{1}$ and $v_{2}$.

\section{THE DYNAMICS}

We consider the motion of two translating masses $\mathcal{Z}$ and $\mathcal{X}$ on a geographical region defined by a closed convex polyhedron with the origin in its interior and not necessarily 
compact. Each boundary of the polyhedron can be associated to a constraint of the form $F^{T} s \leq 1$ denoting the subset of the state space in which the motion is allowed, where $F \in \mathbb{R}^{2}$ is such that $|F|$ characterizes the distance of the boundary $\left\{s \in \mathbb{R}^{2} \mid F^{T} s=1\right\}$ from the origin, while $\frac{F}{|F|}$ is a unit vector orthogonal to the boundary characterizing its orientation, as shown in Figure 1.

Masses $\mathcal{Z}$ and $\mathcal{X}$ move within the geographical region as long as no boundary of the polyhedron is active (namely when the position of a mass is on the boundary and the velocity vector has normal component to the boundary in the same direction as $F$ ). When a boundary is active, the mass motion is not compatible with the direction forbidden by the active boundary and the state is reset to a new value, suitably characterized by the impact of the mass on the active boundary. Figure 1 is illustrative of the setup, where we represented a simple polyhedron defined by one boundary only. We loosely call billiard the geographical region, to enlighten the fact that the dynamics of $\mathcal{Z}$ and $\mathcal{X}$ resembles the behavior of two balls moving on a billiard and impacting on its boundaries.

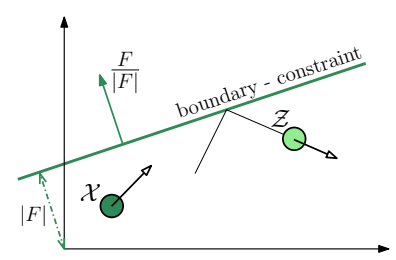

Fig. 1. A closed, convex, not necessarily compact polyhedron with the origin in its interior.

Denoting by $z=\left[\begin{array}{c}z_{p} \\ z_{v}\end{array}\right] \in \mathbb{R}^{4}$ and $x=\left[\begin{array}{l}x_{p} \\ x_{v}\end{array}\right] \in \mathbb{R}^{4}$ respectively the state of $\mathcal{Z}$ and $\mathcal{X}$, the continuous-time dynamics can be given as follows

$$
\begin{gathered}
\mathcal{Z}:\left\{\begin{array}{l}
\dot{z}_{p}=z_{v} \\
\dot{z}_{v}=h(z)
\end{array}\right. \\
\mathcal{X}: \begin{cases}\dot{x}_{p}=x_{v} \\
\dot{x}_{v}=\phi(x)+u\end{cases}
\end{gathered}
$$

where $h, \phi: \mathbb{R}^{4} \rightarrow \mathbb{R}^{2}$ are continuous functions possibly modeling nonlinear factors affecting the acceleration of the translating masses. When a mass impacts a boundary, the position remains unchanged while the velocity $\omega$ is reflected in a direction that is determined by the mass velocity and the boundary orientation. In particular,

$$
\omega^{+}=M(F) \omega
$$

where $M(F)$ is a transformation that inverts only the component of $\omega$ normal to the active boundary, defined by

$$
\begin{aligned}
M(F) & =R(F)^{T} \operatorname{diag}(1,-1) R(F) \\
R(F) & =\frac{1}{|F|}\left[\frac{F^{T}\left[\begin{array}{c}
0-1 \\
1
\end{array}\right]}{F^{T}}\right]
\end{aligned}
$$

where $R(F)$ is the rotation matrix that maps $\omega$ to the base $\left\{w_{\|}, w_{\perp}\right\}=\left\{\left[\begin{array}{cc}0 & 1 \\ -1 & 0\end{array}\right] \frac{F}{|F|}, \frac{F}{|F|}\right\}$, thus decomposing $\omega$ to directions tangential $w_{\|}$and normal $w_{\perp}$ to the boundary; $\left[\begin{array}{cc}1 & 0 \\ 0 & -1\end{array}\right]$ inverts the normal direction $w_{\perp}$, and $R(F)^{-1}=$
$R(F)^{T}$ is the inverse rotation that completes the transformation by mapping $\omega^{+}$to the original base. Note that $\left\langle w_{\|}, w_{\perp}\right\rangle=0$ and $F^{T} w_{\perp}=|F|$.

During impacts the position $\eta$ of the mass remains unchanged, thus $\eta^{+}=\eta$ for $F^{T} \eta=1$ (on the boundary), which can be rewritten in the following form, useful for control design:

$$
\eta^{+}=M(F) \eta+c(F)
$$

where $c(F)=2 \frac{F}{|F|^{2}}$. We establish next this equivalence.

Fact 1: $M(F) \eta+c(F)=\eta$ if and only if $F^{T} \eta=1$.

Based on (4) and Fact 1, the impulsive dynamics of $\mathcal{Z}$ and $\mathcal{X}$ at impacts is summarized by the following equation.

$\mathcal{Z}, \mathcal{X}:\left\{\begin{array}{l}\eta^{+}=M(F) \eta+c(F) \\ \omega^{+}=M(F) \omega\end{array} \quad\right.$ if $F^{T} \eta=1, F^{T} \omega \geq 0$

where the dynamics of $\mathcal{Z}$ arises from using $\eta=z_{p}$ and $\omega=z_{v}$ and the dynamics of $\mathcal{X}$ arises from the identities $\eta=x_{p}$ and $\omega=x_{v}$. For simplicity of exposition, in what follows we will use

$$
\widetilde{M}(F):=\operatorname{diag}(M(F), M(F)), \quad \widetilde{c}(F):=\left[c(F)^{T} \mid 0\right]^{T}
$$

to write compactly the impact model of $\mathcal{Z}$ and $\mathcal{X}$.

The model arising from the combination of continuous motion and impacts is hybrid, meaning that the behavior of the two translating masses cannot be reduced to a continuous motion only (there are discontinuities on the state), or to an impulsive behavior only (there are intervals of time in which the ball moves continuously). In Section IV we will generalize this hybrid dynamics to a polyhedron having $N$ boundaries, using the hybrid system framework of [8], [9], [17], [18]. In particular, we will adopt the notation summarized, e.g., in [9, Section 2.1] (see also [8]), which is not recalled here due to space constraints. For pedagogical reasons, we first present the control design methodology for a geographical region defined by only one boundary. We will consider $\mathcal{Z}$ as an exogenous system that generates a reference trajectory for the controlled system $\mathcal{X}$, which is controlled only during the continuous-time evolution. Thus, loosely speaking, we will consider the goal of finding a control input $u$ that guarantees the asymptotic convergence of the position $x_{p}$ of the controlled system to the position $z_{p}$ of the exogenous system.

\section{TRACKING WITH IMPACTS: SINGLE BOUNDARY}

\section{A. Possible problems of classical approaches}

A naive approach to the solution of the tracking problem is to adopt classical methods for the case without impacts, by defining a control input that enforces asymptotic convergence to zero of the $x-z$ dynamics or, equivalently, asymptotic stability of the set $\mathcal{A}_{\circ}=\{(x, z) \mid x=z\}$ in the absence of impacts. Thus, considering the Lyapunov function $V_{0}$ : $\mathbb{R}^{4} \times \mathbb{R}^{4} \rightarrow \mathbb{R}_{\geq 0}$ defined by

$$
V_{0}(x, z)=(x-z)^{T} P(x-z) .
$$


where $P^{T}=P>0$, for any given $K \in \mathbb{R}^{2 \times 4}$ such that the matrix

$$
A_{c l}=\left[\frac{0_{2 \times 2} \quad I_{2 \times 2}}{K}\right]
$$

satisfies $A_{c l}^{T} P+P A_{c l}<0$, the feedback input

$$
u=h(z)-\phi(x)+K(x-z)
$$

guarantees exponential convergence of $x$ to $z$, as long as impacts never occur. In fact, looking at (1), we have $\dot{V}(x, z)=2(x-z)^{T} P A_{c l}(x-z)<0$ on $\left(\mathbb{R}^{4} \times \mathbb{R}^{4}\right) \backslash \mathcal{A}_{\circ}$.

When impacts (5) are considered, the control law (9) does not anymore guarantee stability nor convergence, as shown in the following example.

Example 1: In Figure 2 the horizontal motion of the two masses $\mathcal{Z}$ and $\mathcal{X}$ is constrained on the left by a wall placed at 0 . The continuous dynamics is given by $\dot{z}=\left[\begin{array}{ll}0 & 1 \\ 0 & 0\end{array}\right] z+\left[\begin{array}{c}0 \\ -\mu\end{array}\right]$ where $\mu>0$ is a constant external force, and $\dot{x}=\left[\begin{array}{ll}0 & 1 \\ 0 & 0\end{array}\right] x+$ $\left[\begin{array}{l}0 \\ 1\end{array}\right] u$ where the input $u=-\mu+[-4-4](x-z)$ guarantees that the matrix $A=\left[\begin{array}{cc}0 & 1 \\ -4 & -4\end{array}\right]$ of the error dynamics $\dot{x}-\dot{z}=$ $A(x-z)$ is Hurwitz.
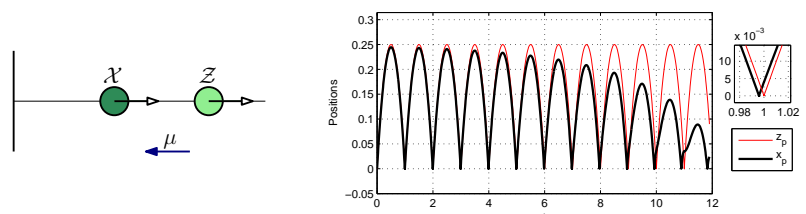

Fig. 2. Example 1: Sketch of the two masses (left); time evolution of the positions of $\mathcal{X}$ and $\mathcal{Z}$ (right).

Given $z_{0}=\left[\begin{array}{ll}0 & v\end{array}\right]^{T}$ and $x_{0}=z_{0}+\varepsilon$, with $\varepsilon \in \mathbb{R}^{2}$ typically small, for a specific set of initial mismatches $\varepsilon$ defined next, the cyclic behavior of the two masses can be qualitatively characterized as a sequence of a continuous motion (where the two masses reverse their direction under the effect of the force $\mu$ ), followed by the impact of $\mathcal{X}$ to the wall, then by the impact of $\mathcal{Z}$, from which this sequence repeats. The mismatch $x-z$ at the $k$ th impact of $\mathcal{Z}$ is given approximately by

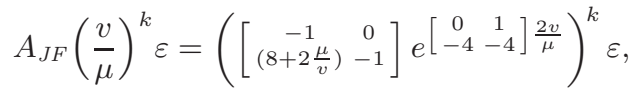

where the matrix $A_{J F}\left(\frac{v}{\mu}\right)$ (whose deduction is given below) presents an unstable eigenvalue when the ratio $\frac{v}{\mu}$ is smaller than 0.613 . For example, given $v=1$ and $\mu=2$, the value of the unstable eigenvalue is 1.34 and the corresponding eigenvector is $\zeta=[0.0773-0.997]^{T}$, thus picking $\varepsilon=\lambda \zeta$, with $0<\lambda \ll 1$, we have that the error $e=x-z$ immediately after the $k$ th impact of $\mathcal{Z}$ is given by $1.34^{k} \varepsilon$, i.e. impacts destabilize the system. Note that the other eigenvalue of $A_{J F}$ has norm less than one, therefore the unstable behavior would appear also for an initial mismatch $\varepsilon$ near $\lambda \zeta, \lambda>0$.

Using $\mu=2$ and $v=1$, consider $e_{0}=x_{0}-z_{0}=\varepsilon \simeq \lambda \zeta$, $0<\lambda \ll 1$. For $v>0$ and $\lambda$ small, the time spent by $\mathcal{Z}$ and $\mathcal{X}$ to go back to zero is given approximately by $\tau_{1}:=\frac{2 v}{\mu}$, and for $t \in\left[0, \tau_{1}\right]$ the time evolution of the error is given by $e(t)=\exp (A t) e_{0}$. Thus, defining $A_{F}\left(\frac{v}{\mu}\right):=\exp \left(A \frac{2 v}{\mu}\right)$, at the time $\tau_{1}^{-}$(immediately before the first impact) we have the error $e\left(\tau_{1}^{-}\right):=A_{F}\left(\frac{v}{\mu}\right) e_{0}=\left[\begin{array}{cc}0.4060 & 0.1353 \\ -0.5413 & -0.1353\end{array}\right] e_{0}$, from which we can also infer that $\mathcal{X}$ impacts first since for $e_{0} \simeq$ $\lambda \zeta, e\left(\tau_{1}^{-}\right) \simeq \lambda\left[\begin{array}{ll}-0.1035 & 0.0931\end{array}\right]^{T}$, that is, $x_{p}\left(\tau_{1}^{-}\right)<z_{p}\left(\tau_{1}^{-}\right)$.

Consider now the interval of time between the impact of $\mathcal{X}$ and the impact of $\mathcal{Z}$ which is given approximately by $\tau_{2}:=-\frac{e_{p}\left(\tau_{1}^{-}\right)}{v}$. When $\mathcal{X}$ impacts, the position does not change, $x_{p}\left(\tau_{1}^{+}\right)=x_{p}\left(\tau_{1}^{-}\right)=0$, while the velocity resets from $x_{v}\left(\tau_{1}^{-}\right)=e_{v}\left(\tau_{1}^{-}\right)+z_{v}\left(\tau_{1}^{-}\right)=e_{v}\left(\tau_{1}^{-}\right)+\left(-v+\mu \tau_{2}\right)=$ $e_{v}\left(\tau_{1}^{-}\right)-\left(v+\frac{\mu}{v} e_{p}\left(\tau_{1}^{-}\right)\right)$to $x_{v}\left(\tau_{1}^{+}\right)=v+\frac{\mu}{v} e_{p}\left(\tau_{1}^{-}\right)-e_{v}\left(\tau_{1}^{-}\right)$, from which we have $e\left(\tau_{1}^{+}\right) \simeq\left[\begin{array}{c}e_{p}\left(\tau_{1}^{-}\right), \\ 2 v+2 \frac{\mu}{v} e_{p}\left(\tau_{1}^{-}\right)-e_{v}\left(\tau_{1}^{-}\right)\end{array}\right]$. The input between the two impacts can be approximated by $u=-\mu+[-4-4] e\left(\tau_{1}^{+}\right) \simeq-\mu-8 v$, from which the error dynamics between the impacts is approximatively given by $\dot{e}_{p}=e_{v}$ and $\dot{e}_{v}=-8 v$. Thus, by integration, at time $\left(\tau_{1}+\tau_{2}\right)^{-}$immediately before the impact of $\mathcal{Z}$, we have $e_{v}\left(\left(\tau_{1}+\tau_{2}\right)^{-}\right)=e_{v}\left(\tau_{1}^{+}\right)-8 v \tau_{2}=2 v+2 \frac{\mu}{v} e_{p}\left(\tau_{1}^{-}\right)-$ $e_{v}\left(\tau_{1}^{-}\right)+8 v \frac{e_{p}\left(\tau_{1}^{-}\right)}{v} \simeq 2 v-e_{v}\left(\tau_{1}^{-}\right)+\left(8+2 \frac{\mu}{v}\right) e_{p}\left(\tau_{1}^{-}\right)$and $e_{p}\left(\left(\tau_{1}+\tau_{2}\right)^{-}\right)=e_{p}\left(\tau_{1}^{+}\right)+e_{v}\left(\tau_{1}^{+}\right) \tau_{2}-4 v \tau_{2}^{2} \simeq-e_{p}\left(\tau_{1}^{-}\right)$, from which $e\left(\left(\tau_{1}+\tau_{2}\right)^{-}\right)=\left[\begin{array}{c}-e_{p}\left(\tau_{1}^{-}\right) \\ 2 v-e_{v}\left(\tau_{1}^{-}\right)+\left(8+2 \frac{\mu}{v}\right) e_{p}\left(\tau_{1}^{-}\right)\end{array}\right]$. From here, $\mathcal{Z}$ impacts and reverses its speed, from which the mismatch is given by $e\left(\left(\tau_{1}+\tau_{2}\right)^{+}\right)=\left[\begin{array}{c}e_{p}\left(\left(\tau_{1}+\tau_{2}\right)^{-}\right) \\ -2 v+e_{v}\left(\left(\tau_{1}+\tau_{2}\right)^{-}\right)\end{array}\right]=$ $\left[\begin{array}{c}-e_{p}\left(\tau_{1}^{-}\right) \\ -e_{v}\left(\tau_{1}^{-}\right)+\left(8+2 \frac{\mu}{v}\right) e_{p}\left(\tau_{1}^{-}\right)\end{array}\right]=A_{J}\left(\frac{\mu}{v}\right) e\left(\tau_{1}^{-}\right)$, where $A_{J}\left(\frac{\mu}{v}\right):=$ $\left[\begin{array}{cc}-1 & 0 \\ 8+2 \frac{\mu}{v} & -1\end{array}\right]$. Finally, after both impacts, the two masses repeat the behavior analyzed above, therefore the mismatch $e$ after the $k$-th impact of $\mathcal{Z}$ can be characterized by $\left(A_{J}\left(\frac{\mu}{v}\right) A_{F}\left(\frac{v}{\mu}\right)\right)^{k} e_{0}=: A_{J F}\left(\frac{v}{\mu}\right)^{k} e_{0}$.

\section{B. Tracking through mirrors}

The defective behavior of Example 1 can be avoided by anticipating the fact that future impacts will invert the (normal) speed of the ball, which can be effectively implemented by tracking the exogenous system $\mathcal{Z}$ through a mirror as shown in Figure 3, where $\mathcal{X}$ may decide to track either the real target $\mathcal{Z}$ or the mirrored target $m(F, \mathcal{Z})$, mirrored through the boundary $F$. Mathematically, the function $m(\cdot, \cdot)$ is given by

$$
m(F, s)=\widetilde{M}(F)(s-\widetilde{c}(F)),
$$

which maps states $z^{+}$after an impact to $m\left(F, z^{+}\right)=z$, as if the impact never happened. Its mirroring peculiarity is underlined by the fact that $m(F, m(F, s))=s$, as stated in the following statement.

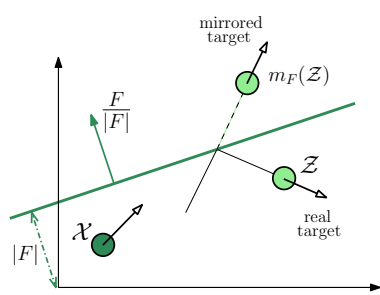

Fig. 3. A possible interpretation of the hybrid tracking algorithm.

Fact 2: $m(F, \widetilde{M}(F) s+\widetilde{c}(F))=s$ and $m(F, m(F, s))=s$. Note that the interaction between the continuous dynamics and the impacts dynamics allows for a nonunique behavior 
of the translating masses from some specific configurations of the state vectors of $\mathcal{X}$ and $\mathcal{Z}$. Indeed, for a geographical region defined by one boundary, $\mathcal{F}=\left\{\left[\begin{array}{l}s_{p} \\ s_{v}\end{array}\right] \in \mathbb{R}^{4} \mid F^{T} s_{p} \leq\right.$ $1\}$, when the state vector characterizes a position $\eta$ on the boundary, $F^{T} \eta=1$, and a velocity $\omega$ with null normal component to the boundary, $F^{T} \omega=0$, both the continuous dynamics and the impacts dynamics can be triggered, with the former enforcing sliding along the boundary while the latter enforcing an infinite sequence of impacts on the boundary (Zeno behavior), each of them resetting the velocity to $F^{T} \omega^{+}=0$. To avoid this kind of phenomena, we restrict the trajectories of $\mathcal{Z}$ to a compact set $\mathcal{K}$ that excludes that set of points, given by

$$
\mathcal{K} \subseteq \mathcal{F} \backslash\left\{\left[\begin{array}{c}
z_{p} \\
z_{v}
\end{array}\right] \in \mathbb{R}^{4} \mid F^{T} z_{p}=1, F^{T} z_{v}=0\right\}
$$

Thus, trajectories of $\mathcal{Z}$ within $\mathcal{K}$ do not present a nonunique behavior, and the same holds for $\mathcal{X}$ as long as its trajectories remain close to the trajectories of $\mathcal{Z}$, as in our local results.

Restricting the state space of $\mathcal{X}$ and $\mathcal{Z}$ to $(x, z) \in \mathcal{C}:=$ $\mathcal{F} \times \mathcal{K}$ and using the mirroring function $m(F, \cdot)$ in $(11)$, the idea of tracking the exogenous system through a mirror can be mathematically characterized by using a new Lyapunov function $V: \mathbb{R}^{4} \times \mathbb{R}^{4} \rightarrow \mathbb{R}_{\geq 0}$,

$$
V(x, z)=\min \left\{V_{0}(x, z), V_{0}(x, m(F, z))\right\},
$$

which extends the quadratic measure of the mismatch between $x$ and $z$ to a combination of the mismatch between $x$ and $z$ and between $x$ and $m(F, z)$, and by casting the tracking problem to the stabilization problem of the set

$$
\mathcal{A}=\left(\mathcal{A}_{\circ} \cup \mathcal{A}_{m}\right) \cap \mathcal{C}
$$

where $\mathcal{A}_{\circ}=\{(x, z) \mid x=z\}$ and $\mathcal{A}_{m}=\{(x, z) \mid x=$ $m(F, z)\}$ intuitively characterize state pairs of $\mathcal{X}$ and $\mathcal{Z}$ that perfectly match (the case of $\mathcal{A}_{\circ}$ ) or that match by looking at the reflection to the boundary/mirror (the case of $\mathcal{A}_{m}$ ). Note that $\mathcal{A}$ is a compact set that can be considered as the generalization of the set $\mathcal{A}_{\circ}$ used in the case without impacts around (7), (9) (the set $\mathcal{A}_{m}$ plays a fundamental role because it allows for the invariance of the set $\mathcal{A}$ along the hybrid dynamics).

The idea is then to asymptotically stabilize the set $\mathcal{A}$ by a feedback $u$ constructed on $x, z$, and $m(F, z)$, and designed to make $V$ decrease along the solutions of the system, namely using the fact that $V$ is a Lyapunov function for $\mathcal{A}$ and the important feature that the stabilization of the set $\mathcal{A}$ is equivalent to the solution of the tracking problem. All these connections are formally stated in the next two lemmas.

Lemma 3.1 (Properties of $V$ ): Given $V$ in (13),

i) $V(x, z)=0$ for each $(x, z) \in \mathcal{A}$,

ii) $V(x, z)>0$ for each $(x, z) \in \mathcal{C} \backslash \mathcal{A}$, and

iii) $V(x, z)=V_{0}(x, z)$ for $(x, z) \in\left(\mathcal{A}_{\circ}+\varepsilon \mathbb{B}\right) \cap \mathcal{C}$, $V(x, z)=V_{0}(x, m(F, z))$ for $(x, z) \in\left(\mathcal{A}_{m}+\varepsilon \mathbb{B}\right) \cap \mathcal{C}$, for $\varepsilon>0$ sufficiently small;

iv) $V \in C^{1}$ on $(\mathcal{A}+\varepsilon \mathbb{B}) \cap \mathcal{C}$, for $\varepsilon>0$ sufficiently small. Lemma 3.1 establishes that $V$ is a candidate Lyapunov function for the set $\mathcal{A}$. Moreover, item iii) above shows that $\min \left(V_{0}(x, z), V_{0}(x, m(F, z))\right)$ is strict in a neighborhood of
$\mathcal{A}$, a feature used in the formulation of the hybrid control law proposed next.

Lemma 3.2 (Property of $\mathcal{A}$ ): If $(x, z) \in \mathcal{A}$, then $x_{p}=z_{p}$

Based on Lemmas 3.1 and 3.2, we can now formulate our hybrid controller for local tracking in the one-boundary case. It will be generalized to the multi-boundary case in Section IV. For the case of one boundary only, and for some specific shapes of the geographical region, the result below can be extended to a global result, as shown in [6].

Theorem 1: Consider $A_{c l}$ in (8) and $V$ in (13), and consider a matrix $P=\left[\begin{array}{l|l}p_{1} I & p_{2} I \\ \hline p_{2} I & p_{3} I\end{array}\right]>0$ with $p_{1}, p_{2}, p_{3} \in$ $\mathbb{R}$, and a matrix gain $K \in \mathbb{R}^{2 \times 4}$ such that for some $\lambda>0$,

$$
A_{c l}^{T} P+P A_{c l}<-\lambda P .
$$

Then the control law:

$u= \begin{cases}h(z)-\phi(x)+K(x-z) & \text { if } \alpha(x, z)<0 \\ M(F) h(z)-\phi(x)+K(x-m(F, z)) & \text { if } \alpha(x, z)>0\end{cases}$

with $\alpha(x, z)=V_{0}(x, z)-V_{0}(x, m(F, z))$, locally asymptotically stabilizes the set $\mathcal{A}$.

The particular structure of $P$ guarantees that $V$ does not increase at jumps. Note that the input differs from the one in (9) only when the function $V(x, z)$ is equal to $V_{0}(x, m(F, z))$, that is, intuitively, when the mirrored target $m(F, z)$ is closer to $x$ than the real target $z$. Note also that $u$ is not defined for $V_{0}(x, z)=V_{0}(x, m(F, z))$ but this is not an issue in a small neighborhood of $\mathcal{A}$, as shown in Lemma 3.1. Finally, the notion of pre-asymptotic stability used in Theorem 1 underlines the fact that some solutions may have a compact domain, since the motion of $\mathcal{X}$ and $\mathcal{Z}$ is restricted to $\mathcal{C}$.

Theorem 1 can be established by applying hybrid Lyapunov and LaSalle-like tools [17], [8] to the following hybrid system, which models the interaction between $\mathcal{X}$ and $\mathcal{Z}$ :

$$
\begin{aligned}
& \left\{\begin{array}{l}
\dot{x}_{p}=z_{v} \\
\dot{x}_{v}=\phi(x)+u \quad(x, z) \in C_{x} \times C_{z} \\
\dot{z}_{p}=z_{v} \\
\dot{z}_{v}=h(z)
\end{array}\right. \\
& \left\{\begin{array}{l}
x^{+}=\widetilde{M}(F) x+\widetilde{c}(F) \quad(x, z) \in D_{x} \times C_{z} \\
z^{+}=z \\
x^{+}=x \\
z^{+}=\widetilde{M}(F) z+\widetilde{c}(F) \quad(x, z) \in C_{x} \times D_{z}
\end{array}\right.
\end{aligned}
$$

where $C_{\mathcal{X}}=\mathcal{F}, C_{\mathcal{Z}}=\mathcal{K}, D_{\mathcal{X}}=\mathcal{F} \cap\left\{x \mid F^{T} x_{p}=1, F^{T} x_{v} \geq\right.$ $0\}$, and $D_{\mathcal{Z}}=\mathcal{K} \cap\left\{x \mid F^{T} z_{p}=1, F^{T} z_{v} \geq 0\right\}$.

Note that an impact of both $\mathcal{X}$ and $\mathcal{Z}$ at the same time instant is modeled by a sequence of two consecutive jumps, one for each system. This feature is not restrictive, since the sequence of jumps is characterized by hybrid time instants $(t, j),(t, j+1)$ and $(t, j+1),(t, j+2)$, thus both at the same ordinary time $t$.

Using the hybrid dynamics in (17) (note that $\mathcal{C}=C_{\mathcal{X}} \cup$ $C_{\mathcal{Z}}$ ), the proof of Theorem 1 follows from the combination of the invariance principle in [17] and the following lemma, which shows that $V$ decreases along the continuous motion of the two translating masses (i.e. along flows of $\mathcal{H}$ ) and 
it does not increases on impacts (i.e. across jumps), from which it is also possible to partially justify the importance of avoiding Zeno solutions.

Lemma 3.3: Under the hypotheses of Theorem 1, for each $(x, z) \in \mathcal{C}$,

- $\dot{V}(x, z) \leq-\lambda V(x, z)$ if $\alpha(x, z) \neq 0$,

- $V\left(x^{+}, z^{+}\right) \leq V(x, z)$ if $(x, z) \in\left(C_{x} \times D_{z}\right) \cup\left(D_{x} \times C_{z}\right)$.

Thus, combining Theorem 1 and Lemma 3.2, we get the following result.

Corollary 1: The control $u$ in (16) guarantees that (i) for each $\varepsilon>0$ there exists $\delta>0$ such that $|(x, z)|_{\mathcal{A}} \leq \delta$ implies $\left|x_{p}(t, j)-z_{p}(t, j)\right| \leq \varepsilon$ for all $(t, j) \in \operatorname{dom}(x, z)$, and (ii) there exists $\gamma>0$ such that for each complete solution ${ }^{1}$ $(x, z)$ from $\left|\left(x_{0}, z_{0}\right)\right|_{\mathcal{A}} \leq \gamma, \lim _{t+j \rightarrow \infty}\left|x_{p}(t, j)-z_{p}(t, j)\right|=0$. Finally, for implementation reasons, in the next proposition we propose a specific but constructive solution to the inequality (15).

Proposition 1 (Feasibility): Select $k_{1}, k_{2} \in \mathbb{R}$ such that $\bar{A}=\left[\begin{array}{cc}0 & 1 \\ -k_{1} & -k_{2}\end{array}\right]$ is a Hurwitz matrix, and take $\bar{P}=\left[\begin{array}{ll}p_{1} & p_{2} \\ p_{2} & p_{3}\end{array}\right] \in$ $\mathbb{R}^{2}$ solution to $\bar{A}^{T} \bar{P}+\overline{P A}<0$. Then, (15) is satisfied by

$$
P=\left[\begin{array}{l|l}
p_{1} I & p_{2} I \\
\hline p_{2} I & p_{3} I
\end{array}\right], K=-\left[\begin{array}{l|l}
k_{1} I \mid k_{2} I
\end{array}\right] .
$$

\section{LOCAL TRACKING WITH MANY BOUNDARIES}

A geographical region defined by a polyhedron with many boundaries is given by

$$
\mathcal{F}=\left\{\left[\begin{array}{c}
s_{p} \\
s_{v}
\end{array}\right] \in \mathbb{R}^{4} \mid \forall k \in \mathcal{I}, F_{k}^{T} s_{p} \leq 1\right\}
$$

where each $F_{k} \in \mathbb{R}^{2}$ characterizes a boundary $\mathcal{F}_{k}=\left\{\left[\begin{array}{c}s_{p} \\ s_{v}\end{array}\right] \in\right.$ $\left.\mathbb{R}^{4} \mid F_{k}^{T} s_{p}=1\right\}$, and $\mathcal{I}=\{1, \ldots, N\}, N \in \mathbb{N}$, is an index set. From Section III, we recover the assumption on the state-space of $\mathcal{X}$ and $\mathcal{Z}$ that guarantees the absence of the "sliding vs impacts" phenomena characterized by (12). Mathematically, with many boundaries, the corresponding set of defective points to be excluded is given by

$$
\mathcal{Q}_{1}=\bigcup_{k \in \mathcal{I}}\left(\left\{\left[\begin{array}{l}
s_{p} \\
s_{v}
\end{array}\right] \in \mathbb{R}^{4} \mid F_{k}^{T} s_{p}=1, F_{k}^{T} s_{v}=0\right\}\right)
$$

Another source of nonuniqueness in the behavior of $\mathcal{X}$ and $\mathcal{Z}$ is given by corners points (namely any point $s \in \mathcal{F}_{k} \cap \mathcal{F}_{j}$ for $k, j \in \mathcal{I}$ and $k \neq j$ ). Excluding these points is important for the feasibility of the tracking algorithm. In fact, suppose that $\mathcal{X}$ and $\mathcal{Z}$ impact together the point $s$ at the intersection two boundaries, $k$ and $j$. Then, nondeterministically, $\mathcal{X}$ may follow the impact dynamics enforced by $k$, while $\mathcal{Z}$ may follow the one from $j$, thus showing that a solution to the tracking problem cannot be achieved. Mathematically, this set of points is given by

$$
\mathcal{Q}_{2}=\bigcup_{i, j \in \mathcal{I}}\left\{\left[\begin{array}{l}
s_{p} \\
s_{v}
\end{array}\right] \in \mathbb{R}^{4} \mid F_{i}^{T} s_{p}=F_{j}^{T} s_{p}=1, i \neq j\right\}
$$

Thus, we restrict the trajectories of $\mathcal{Z}$ to the compact set

$$
\mathcal{K} \subset \mathcal{F} \backslash\left(\mathcal{Q}_{1} \cup \mathcal{Q}_{2}\right) .
$$

\footnotetext{
${ }^{1}$ Namely, solutions whose hybrid time domain is unbounded.
}

The hybrid system $\mathcal{H}$ in (17) can be generalized to the geographical region (19) by introducing the set $\mathcal{D}_{k}=$ $\left\{\left[\begin{array}{l}s_{p} \\ s_{v}\end{array}\right] \in \mathbb{R}^{4} \mid F_{k}^{T} s_{p}=1, F_{k}^{T} s_{v} \geq 0\right\}$, based on which, the flow and jump sets of $\mathcal{X}$ and $\mathcal{Z}$ can be defined as $C_{\mathcal{X}}=\mathcal{F}$, $C_{\mathcal{Z}}=\mathcal{K}, D_{\mathcal{X}}=\mathcal{F} \cap\left\{\left[\begin{array}{l}x_{p} \\ x_{v}\end{array}\right] \mid \exists k \in \mathcal{I}, x \in \mathcal{D}_{k}\right\}$, and $D_{\mathcal{Z}}=\mathcal{K} \cap\left\{\left[\begin{array}{c}z_{p} \\ z_{v}\end{array}\right] \mid \exists k \in \mathcal{I}, z \in \mathcal{D}_{k}\right\}$, where $D_{\mathcal{X}}$ and $D_{\mathcal{Z}}$ are essentially defined by the intersection of $C_{\mathcal{X}}$ and $C_{\mathcal{Z}}$ with the impact points. Thus, with these flow and jump sets, the hybrid model $\mathcal{H}$ can be defined by the flow dynamics (17a) and the following jump dynamics:

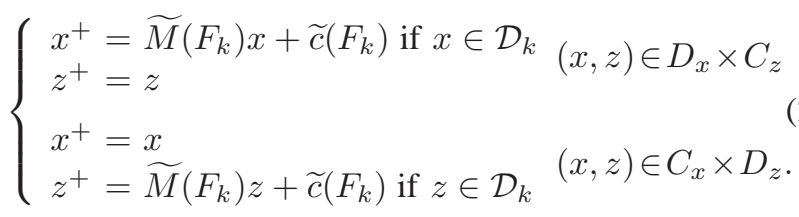

Note that the set $\mathcal{C}=C_{x} \times C_{z}$ is now generated by the many boundary geographical region $\mathcal{F}$ in (19) and by the compact set $\mathcal{K}$ in (22). Moreover, to simplify the notation, we define the set $\mathcal{D}=\left(D_{x} \times C_{z}\right) \cup\left(C_{x} \times D_{z}\right)$ characterizing the set of points from which an impact may occur, and we rewrite the mirroring function $m(\cdot, \cdot)$ and the impact matrix $M(F)$ in the following form

$$
\begin{aligned}
m\left(F_{k}, s\right) & = \begin{cases}s & \text { if } k=0 \\
\widetilde{M}\left(F_{k}\right)\left(s-\widetilde{c}\left(F_{k}\right)\right) & \text { if } k \in \mathcal{I}\end{cases} \\
M\left(F_{k}\right) & = \begin{cases}I & \text { if } k=0 \\
M\left(F_{k}\right) & \text { if } k \in \mathcal{I},\end{cases}
\end{aligned}
$$

and the Lyapunov function $V$ can now be defined as

$$
V(x, z)=\min _{k \in\{0\} \cup \mathcal{I}} V_{0}\left(x, m\left(F_{k}, z\right)\right) .
$$

with the closest mirrored target defined by

$$
\sigma=\underset{k \in\{0\} \cup \mathcal{I}}{\operatorname{argmin}} V_{0}\left(x, m\left(F_{k}, z\right)\right) .
$$

where $\sigma=0$ denotes the real target. Note that $\sigma$ is only well defined in regions where the minimum in (25) is strict. One such region is characterized in Item iii) of Lemma 4.1, below. In analogy with the previous section, the set $\mathcal{A}$ can now be generalized to

$$
\begin{aligned}
\mathcal{A} & =\left(\bigcup_{k \in\{0\} \cup \mathcal{I}} \mathcal{A}_{k}\right) \cap \mathcal{C} \\
\mathcal{A}_{k} & =\left\{(x, z) \in \mathbb{R}^{4} \times \mathbb{R}^{4} \mid x=m\left(F_{k}, z\right)\right\},
\end{aligned}
$$

and the connections between the Lyapunov function $V$ in (25), the set $\mathcal{A}$ in (27), and the tracking problem are presented in the next two lemmas.

Lemma 4.1 (Properties of $V$ ): Given $V$ in (25),

i) $V(x, z)=0, \forall(x, z) \in \mathcal{A}$.

ii) $V(x, z)>0, \forall(x, z) \in \mathcal{C} \backslash \mathcal{A}$.

iii) There exists $\varepsilon>0$ s.t. $\forall(x, z) \in\left(\mathcal{A}_{k}+\varepsilon \mathbb{B}\right) \cap \mathcal{C}, \sigma=k$.

iv) There exists $\varepsilon>0$ s.t. $V \in C^{1}$ on $(\mathcal{A}+\varepsilon \mathbb{B}) \cap \mathcal{C}$.

Lemma 4.2 (Property of $\mathcal{A}$ ): If $(x, z) \in \mathcal{A}$, then $x_{p}=z_{p}$. From Lemmas 4.1 and 4.2, we can focus on the stabilization of the set $\mathcal{A}$, using the function $V$ as a candidate Lyapunov function. Thus, the control law (16) can be generalized to the many boundaries case as in the following theorem. 
Theorem 2: Consider $\mathcal{H}$ in (17a), (23). Suppose that a matrix $P=\left[\begin{array}{l|l}p_{1} I & p_{2} I \\ \hline p_{2} I & p_{3} I\end{array}\right]>0, p_{1}, p_{2}, p_{3} \in \mathbb{R}$, and a gain matrix $K \in \mathbb{R}^{2 \times 4}$ satisfy equation (15) with $\mathcal{A}_{c l}$ in (8) and $\lambda>0$. Then the control law:

$$
u=M\left(F_{\sigma}\right) h(z)-\phi(x)+K\left(x-m\left(F_{\sigma}, z\right)\right),
$$

with $M\left(F_{\sigma}\right)$ and $m\left(F_{\sigma}, z\right)$ as in (24), (26) is well defined in a neighborhood of $\mathcal{A}$ and it locally asymptotically stabilizes the set $\mathcal{A}$.

Theorem 2 can be established from Lemma 4.1, the invariance principle in [16] and Lemma 4.3 below. Moreover, by Lemma 4.2, Theorem 2 entails asymptotic convergence of $x_{p}$ to $z_{p}$, paralleling Corollary 1 of previous section.

Lemma 4.3: Using the aggregate state $\xi=(x, z)$, denoting by $H(\xi)$ the right-hand side of the flow map (17a) and by $G(\xi)$ the right-hand side of the the jump map (23), under the hypothesis of Theorem 2, the control law (28) guarantees that there exists $\lambda \in(0,1)$ and $\varepsilon>0$ sufficiently small such that

$$
\begin{aligned}
\langle\nabla V(\xi), H(\xi)\rangle<-\lambda V(\xi) & \forall \xi \in((\mathcal{A}+\varepsilon \mathbb{B}) \cap \mathcal{C}) \backslash \mathcal{A} \\
V(g) \leq V(\xi) & \forall \xi \in(\mathcal{A}+\varepsilon \mathbb{B}) \cap \mathcal{D}, \forall g \in G(\xi) .
\end{aligned}
$$

\section{Simulation examples}

We consider a billiard with five boundaries defined by

$$
\left[F_{1}\left|F_{2}\right| F_{3}\left|F_{4}\right| F_{5}\right]=\left[\begin{array}{c|c|c|c|c}
0 & -1 & -1 & \frac{1}{2} & \frac{3}{4} \\
-1 & 0 & 1 & 1 & -1
\end{array}\right]
$$

and a simple dynamics for $\mathcal{Z}$ given by $\dot{z}_{p}=z_{v}, \dot{z}_{v}=0$. Using $P$ and $K$ given by

$$
P=\left[\begin{array}{l|l}
0.4 I & 0.1 I \\
\hline 0.1 I & 0.2 I
\end{array}\right] \text { and } K=-[10 I \mid 11 I],
$$

in Figure 4 we show a comparison between the classical control law (9) and the hybrid control law (28). The two upper rows of Figure 4 represent trajectories of $\mathcal{X}$ and $\mathcal{Z}$ on the billiard from $z_{0}=\left[\begin{array}{llll}0.8 & 0.4 & 1.5 & 1.25\end{array}\right]^{T}$ and $x_{0}=$ [ $\left.\begin{array}{lll}0.6 & 1.5 & 1.25\end{array}\right]$, for simulation times $T=3.2$. Finally, even though Theorem 2 only guarantees local properties, the good behavior induced by the proposed approach for large initial errors is reported in the third row of Figure 4. Note that (28) is undefined where the minimum of (25) is nonstrict. But since this is a set of measure zero, this is not an issue for running this simulation. A regularization of (28) to make it well defined everywhere in $\mathcal{K}$ is carried out in the companion paper [6], which focuses on global tracking.

\section{REFERENCES}

[1] J.M. Bourgeot and B. Brogliato. Tracking control of complementarity Lagrangian systems. Int. J. Bifurcation Chaos, 15(6):1839-1866, 2005.

[2] B. Brogliato. Nonsmooth mechanics: models, dynamics, and control. Springer Verlag, 1999.

[3] B. Brogliato. Absolute stability and the Lagrange-Dirichlet theorem with monotone multivalued mappings. Systems \& control letters, 51(5):343-353, 2004.

[4] B. Brogliato, S. I. Niculescu, and P. Orthant. On the control of finitedimensional mechanical systems with unilateral constraints. IEEE Transactions on Automatic Control, 42(2):200-215, 1997.

[5] M. Buhler, D.E. Koditschek, and P.J. Kindlmann. A family of robot control strategies for intermittent dynamical environments. IEEE Control Systems Magazine, 10(2):16-22, 1990.
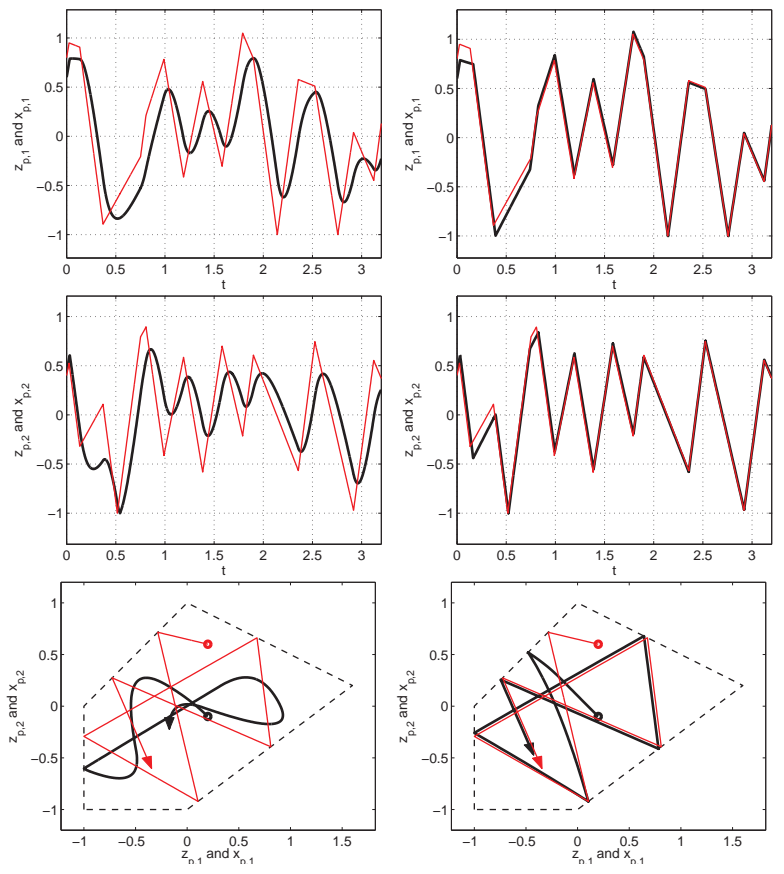

Fig. 4. Positions of $\mathcal{Z}$ (light/red), $\mathcal{X}$ (dark/black). Left: classical control law (9). Right: hybrid control law (28).

[6] F. Forni, A.R. Teel, and L. Zaccarian. Tracking control in billiards using mirrors without smoke, part II: additional Lyapunov-based local and global results. In Joint CDC-ECC, Orlando (FL), USA, December 2011, to appear.

[7] S. Galeani, L. Menini, A. Potini, and A. Tornambè. Trajectory tracking for a particle in elliptical billiards. Int. J. of Control, 81(2):189-213, February 2008.

[8] R. Goebel, R. Sanfelice, and A.R. Teel. Hybrid dynamical systems. Control Systems Magazine, IEEE, 29(2):28-93, April 2009.

[9] R. Goebel and A.R. Teel. Solutions to hybrid inclusions via set and graphical convergence with stability theory applications. Automatica, 42(4):573 - 587, 2006

[10] R.I. Leine and N. van de Wouw. Stability and convergence of mechanical systems with unilateral constraints. Lecture Notes in Applied and Computational Mechanics. Springer Verlag, 2008.

[11] R.I. Leine and N. van de Wouw. Uniform convergence of monotone measure differential inclusions: with application to the control of mechanical systems with unilateral constraints. International Journal of Bifurcation and Chaos in Applied Sciences and Engineering, 18(5):1435-1457, 2008.

[12] L. Menini and A. Tornambè. Asymptotic Tracking of Periodic Trajectories for a Simple Mechanical System Subject to Nonsmooth Impacts. IEEE Trans. Aut. Control, 46:1122-1126, 2001.

[13] I.C. Morarescu and B. Brogliato. Trajectory tracking control of multiconstraint complementarity Lagrangian systems. IEEE Trans. on Aut. Control, 55(6):1300-1313, 2010.

[14] PR Pagilla. Control of contact problem in constrained Euler-Lagrange systems. IEEE Trans. Aut. Cont., 46(10):1595-1599, 2001.

[15] PR Pagilla and B. Yu. An experimental study of planar impact of a robot manipulator. IEEE/ASME Transactions on Mechatronics, 9(1):123-128, 2004.

[16] R.G. Sanfelice. Robust Hybrid Control Systems. PhD thesis, University of California, Santa Barbara, 2007.

[17] R.G. Sanfelice, R. Goebel, and A.R. Teel. Invariance principles for hybrid systems with connections to detectability and asymptotic stability. IEEE Transactions on Automatic Control, 52(12):2282-2297, 2007.

[18] R.G. Sanfelice, R. Goebel, and A.R. Teel. Generalized solutions to hybrid dynamical systems. ESAIM: COCV, 14(4):699-724, October 2008.

[19] P. Sekhavat, N. Sepehri, and Q. Wu. Impact stabilizing controller for hydraulic actuators with friction: Theory and experiments. Control Engineering Practice, 14(12):1423-1433, 2006.

[20] A. Tornambe. Modeling and control of impact in mechanical systems: Theory and experimental results. IEEE Trans. Aut. Cont., 44(2):294309, 1999. 